\title{
Intradural spinal tumours and their mimics: a review of radiographic features
}

\author{
Sara Wein, Francesco Gaillard
}

Department of Radiology, Royal Melbourne Hospital, Parkville, Victoria, Australia

\section{Correspondence to} Dr Sara Wein, Radiology Department, Royal Melbourne Hospital, Grattan Street, Parkville, VIC 3050, Australia; sarawein@bigpond.com

Received 6 December 2012 Revised 5 May 2013 Accepted 14 May 2013 Published Online First 7 June 2013

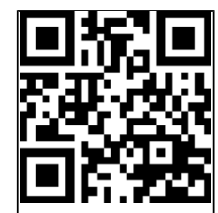
Editor's choice can to access mo free content

To cite: Wein S, Gaillard F. Postgrad Med J

2013;89:457-469.

\begin{abstract}
Intradural spinal tumours, although relatively uncommon, can be diagnostically challenging, and often result in significant morbidity. They can be subdivided according to their cell of origin and whether they are within the cord (intramedullary) or intradural but extramedullary in location. The differential diagnosis for masses of the cauda equina region is often considered separately. Additionally, some inflammatory processes, cysts, benign tumour-like masses and vascular malformations may mimic intradural tumours. Although in many instances, a precise preoperative diagnosis is not possible as many of the imaging findings overlap, some features may strongly suggest one diagnosis over others. This article reviews the range of intradural spinal tumours in the adult and paediatric populations, with an emphasis on pertinent imaging characteristics. An approach is provided for distinguishing tumours from lesions that mimic tumours and for narrowing the differential diagnosis according to imaging findings.
\end{abstract}

\section{INTRODUCTION}

Intradural spinal tumours can be diagnostically challenging and often result in significant morbidity. Although relatively uncommon compared with intracranial or extradural spinal masses, the need for a preoperative or non-operative diagnosis is in many ways greater, as biopsy of intradural lesions has the potential to cause devastating neurological impairment. Additionally, as the presentation of intradural spinal tumours is similar for all histologies, being dependent on tumour size and location, clinical features are often unhelpful in narrowing the differential. The most common presenting symptoms include back or neck pain, radicular pain, weakness, paraesthesia, gait disturbance and bowel and bladder dysfunction. Brown-Sequard syndrome (ipsilateral paralysis and loss of proprioception, and contralateral loss of pain and temperature sensation) due to compression of one side of the spinal cord and acute headache due to subarachnoid haemorrhage are less common presenting features. ${ }^{1}$

Intradural tumours can be subdivided according to their location into intramedullary and intradural extramedullary tumours. The cauda equina region is often considered separately to the remainder of the spinal cord as certain tumours are particular to it. Additionally, a number of non-neoplastic lesions may mimic intramedullary and intradural extramedullary tumours. This article reviews the range of intradural spinal tumours in the adult and paediatric populations, with an emphasis on pertinent imaging characteristics. An approach is provided for distinguishing tumours from lesions that mimic tumours and for narrowing the differential diagnosis according to imaging findings, thus allowing the formulation of an appropriate management plan.

\section{IMAGING OF INTRADURAL MASS LESIONS}

MRI is the modality of choice for the assessment of lesions within the spinal canal as it has exquisite contrast and structural resolution, is able to image all compartments, and is able to assess for the presence of enhancement, cystic change and blood product. Myelography, historically, was of prime importance, but is now routinely used only in patients for whom MRI is contraindicated, or occasionally as a problem-solving technique. It is usually combined with CT (ie, CT myelography). CT remains the best modality for assessing the osseous structures and is especially important in planning instrumentation. Angiography is useful in a select group of patients who have vascular tumours or vascular malformations, and may offer endovascular therapeutic options. Ultrasound has been shown to be valuable in the assessment of spinal tumours in newborns and young infants, however, does not have a role in older patients as it is unable to image the intradural compartment due to the overlying posterior spinal elements. ${ }^{2}$

\section{INTRAMEDULLARY TUMOURS}

Intramedullary spinal tumours represent $4-10 \%$ of all central nervous system (CNS) tumours. ${ }^{3}$ They account for $20 \%$ of all intradural tumours in adults, and $35 \%$ of all intradural tumours in children (box 1). ${ }^{1}$ The vast majority $(95 \%)$ are glial tumours. ${ }^{4}$

Three general characteristics of intramedullary neoplasms are recognised on MRI: they cause spinal cord expansion, they produce high signal intensity on T2 weighted images, and the majority show at least some contrast enhancement. Intramedullary tumours are also commonly

\section{Box 1 Intramedullary tumours (20\% in} adults, $35 \%$ in children)

- Ependymoma $(60 \%)$

- Astrocytoma $(30 \%)$

- Hemangioblastoma

- Ganglioglioma

- Intramedullary metastasis

- Primary intramedullary lymphoma

- Primitive neuroendocrine tumour

- Solitary fibrous tumour 
associated with cysts and syringohydromyelia, and may have evidence of prior haemorrhage.

\section{Ependymoma}

Ependymomas are the most common intramedullary neoplasm in adults and the most common intramedullary tumour overall, comprising approximately $60 \%$ of all glial spinal cord tumours, and occurring in approximately 0.21 per 100000 persons per year. ${ }^{4} 5$ They represent $30 \%$ of paediatric intramedullary neoplasms, making them the second most common paediatric intramedullary neoplasm, after astrocytomas. ${ }^{3}$ Although most are sporadic, there is an increased incidence in neurofibromatosis type 2 (NF2).

The majority are WHO grade II lesions, however, anaplastic grade III lesions are encountered. They are generally slow growing and tend to compress adjacent spinal cord tissue rather than infiltrate it, almost always leaving a cleavage plane between tumour and spinal cord tissue.

\section{Radiographic features}

Ependymomas can occur anywhere along the spinal cord, however, the cervical cord is the most common site. ${ }^{6}$ The myxopapillary variant almost exclusively appears as an extramedullary mass in the region of the cauda equina and is discussed separately.
As ependymomas arise from ependymal cells lining the central canal, they tend to occupy the central portion of the spinal cord and cause symmetric cord expansion (figure 1). Although unencapsulated, they are well circumscribed, and are frequently associated with cysts (tumoral and non-tumoral) and syringohydromyelia. In contrast with intracranial ependymomas, calcification is uncommon. Most ependymomas are isointense to hypointense on T1, however, mixed signal may be seen if cyst formation, tumour necrosis or haemorrhage has occurred. Virtually all enhance strongly. They are typically hyperintense on T2, and most demonstrate peritumoural oedema. ${ }^{4}$ Associated haemorrhage leads to a hypointense haemosiderin rim above and/or below the mass ('cap sign') in approximately one-third of cases. ${ }^{4}$ The cap sign is suggestive of but not pathognomonic of ependymoma, as it may also be seen in haemangioblastomas and paragangliomas. Scoliosis and bony remodelling may occur and are more commonly seen in association with ependymomas than astrocytomas (table 1).

\section{Astrocytoma}

Astrocytomas are the second most common intramedullary tumour overall, representing approximately $40 \%$ of such lesions and occurring in approximately 0.03 per 100000 persons per year. $^{5}{ }^{6}$ They are associated with neurofibromatosis type 1 (NF1). ${ }^{6}$ They generally have a lower histologic grade than

Figure 1 Ependymoma. (A) and (B) T2 weighted images demonstrate a heterogeneous mass located centrally, displacing normal cord laterally (white arrow heads) with associated peritumoural cysts/syrinx (white arrow) and prominent inferior haemosiderin capping (black arrow) (C) T1 weighted. (D) T1 weighted with gadolinium demonstrating prominent tumoural enhancement. 
Table 1 MRI features of intradural tumours

\begin{tabular}{|c|c|c|c|c|c|c|}
\hline & Most common location & T1 & $\mathrm{T} 2$ & Enhancement & Morphology & Special features \\
\hline \multicolumn{7}{|l|}{ Intramedullary tumours } \\
\hline Ependymoma & Cervical cord & $\begin{array}{l}\text { Isointense to } \\
\text { hypointense }\end{array}$ & hyperintense & Strong & $\begin{array}{l}\text { Well-circumscribed. } \\
\text { Central within cord }\end{array}$ & $\begin{array}{l}\text { Cysts and syringohydromyelia common } \\
\text { Haemosiderin cap sign }\end{array}$ \\
\hline Astrocytoma & Thoracic cord & $\begin{array}{l}\text { Isointense to } \\
\text { hypointense }\end{array}$ & Hyperintense & Variable and patchy & $\begin{array}{l}\text { Poorly defined margins } \\
\text { Eccentric within cord }\end{array}$ & May involve long cord segments \\
\hline Hemangioblastoma & Thoracic cord & $\begin{array}{l}\text { Isointense to } \\
\text { hypointense }\end{array}$ & $\begin{array}{l}\text { Isointense to } \\
\text { hyperintense }\end{array}$ & Vivid & Usually discrete nodules & $\begin{array}{l}\text { Cysts and syrinx common } \\
\text { Flow voids }\end{array}$ \\
\hline Ganglioglioma & Cervical cord & Heterogeneous & Hyperintense & Patchy & Eccentric within cord & $\begin{array}{l}\text { Calcification common } \\
\text { Cysts common }\end{array}$ \\
\hline Intramedullary metastases & Cervical cord & Hypointense & Hyperintense & Vivid & Well defined & Prominent peritumoural oedema \\
\hline Primary intramedullary lymphoma & Cervical cord & isointense & Hyperintense & Homogeneous & Poorly defined & \\
\hline Primitive neuroendocrine tumour & $\begin{array}{l}\text { Cauda equina/filum } \\
\text { terminale }\end{array}$ & Hypointense & Hyperintense & Heterogeneous & Poorly defined & $\begin{array}{l}\text { Leptomeningeal metastases/enhancement } \\
\text { common }\end{array}$ \\
\hline Solitary fibrous tumour & & $\begin{array}{l}\text { Isointense to } \\
\text { hypointense }\end{array}$ & Markedly hypointense & Vivid & Well circumscribed & \\
\hline \multicolumn{7}{|l|}{ Intradural extramedullary tumours } \\
\hline Schwannoma & Cervical cord & $\begin{array}{l}\text { Isointense to } \\
\text { hypointense }\end{array}$ & Hyperintense & $\begin{array}{l}\text { May be } \\
\text { heterogeneous }\end{array}$ & Round, sausage or dumbbell shaped & $\begin{array}{l}\text { Bony remodelling common } \\
\text { Haemorrhage, cysts and fatty degeneration may } \\
\text { occur } \\
\text { Displace nerve roots }\end{array}$ \\
\hline Meningioma & Thoracic cord & $\begin{array}{l}\text { Isointense to } \\
\text { hypointense }\end{array}$ & $\begin{array}{l}\text { Isointense to } \\
\text { hyperintense }\end{array}$ & Vivid diffuse & Well circumscribed & $\begin{array}{l}\text { Dural tail } \\
\text { May calcify }\end{array}$ \\
\hline Neurofibroma & Cervical cord & Hypointense & Hyperintense & Heterogeneous & Fusiform & $\begin{array}{l}\text { Bony remodelling common } \\
\text { Encase nerve roots } \\
\text { Target sign }\end{array}$ \\
\hline Myxopapillary ependymoma & $\begin{array}{l}\text { Cauda equina/ filum } \\
\text { terminale }\end{array}$ & Usually hypointense & Hyperintense & $\begin{array}{l}\text { Usually } \\
\text { homogeneous }\end{array}$ & Well circumscribed & Haemorrhage common \\
\hline Paraganglioma & $\begin{array}{l}\text { Cauda equina/filum } \\
\text { terminale }\end{array}$ & Isointense & Hyperintense & Intense & Well circumscribed & $\begin{array}{l}\text { Flow voids } \\
\text { Cap sign } \\
\text { Salt-and-pepper appearance }\end{array}$ \\
\hline Leptomeningeal metastases & Lumbosacral spine & Isointense & $\begin{array}{l}\text { Isointense to } \\
\text { hyperinense }\end{array}$ & Smooth or nodular & $\begin{array}{l}\text { Thickened nerve roots or nodular } \\
\text { lesions }\end{array}$ & $\begin{array}{l}\text { Sugar coating } \\
\text { Cord oedema may be present }\end{array}$ \\
\hline
\end{tabular}


astrocytomas in the brain, with approximately $75 \%$ being either grade I or II lesions. ${ }^{4}$

Spinal cord astrocytomas account for $60 \%$ of paediatric intramedullary tumours, making them the most common spinal cord tumour in children. ${ }^{3}$ Pilocytic astrocytomas are a subtype of astrocytoma found predominantly in children and young adults. They are WHO grade I lesions and are associated with an excellent prognosis, as they behave much like grade I cerebellar pilocytic astrocytomas and displace neural tissue rather than infiltrate it.

Diffuse astrocytomas are generally faster growing than ependymomas and typically have a worse prognosis. They are characterised by hypercellularity and the absence of a surrounding capsule and, in contrast with cord ependymomas, a cleavage plane is not present. Surgical excision is usually the treatment of choice, however, due to their infiltrative nature, resection is almost always histologically incomplete.

\section{Radiographic features}

The most common location of astrocytomas is the thoracic cord, followed by the cervical cord. Isolated involvement of the conus medullaris and filum terminale is rare. ${ }^{4}$

Astrocytomas are typically long intramedullary masses that cause diffuse cord expansion. Involvement of the entire spinal cord (holocord presentation) may occur. It is more common in children than in adults, and is more frequent with pilocytic astrocytomas.
As astrocytomas arise from cord parenchyma, they typically have an eccentric location (figure 2) and may be exophytic, sometimes even appearing largely extramedullary. They usually have poorly defined margins and, like ependymomas, are typically isointense to hypointense on $\mathrm{T} 1$ with variable and patchy contrast enhancement. They are hyperintense on T2 Haemorrhage is uncommon, and associated peritumoural oedema and cysts are less prominent than with ependymomas.

\section{Hemangioblastoma}

Hemangioblastomas are the third most common intramedullary spinal neoplasm, representing $2 \%$ of such tumours and occurring in approximately 0.02 per 100000 persons per year. ${ }^{7}$ Two-thirds are sporadic, with a peak presentation in the fourth decade. One-third of patients have von Hippel-Lindau syndrome. ${ }^{8}$ These patients typically present earlier and with multiple tumours.

Haemangioblastomas are vascular WHO grade I lesions and are usually treated by surgical resection, sometimes with preceding endovascular embolisation to reduce intraoperative blood loss.

\section{Radiographic features}

The most common location is the thoracic cord. Although they usually appear as discrete nodules, there can be diffuse cord expansion. They have a variable appearance on T1, however, the majority are isointense to hypointense and difficult to

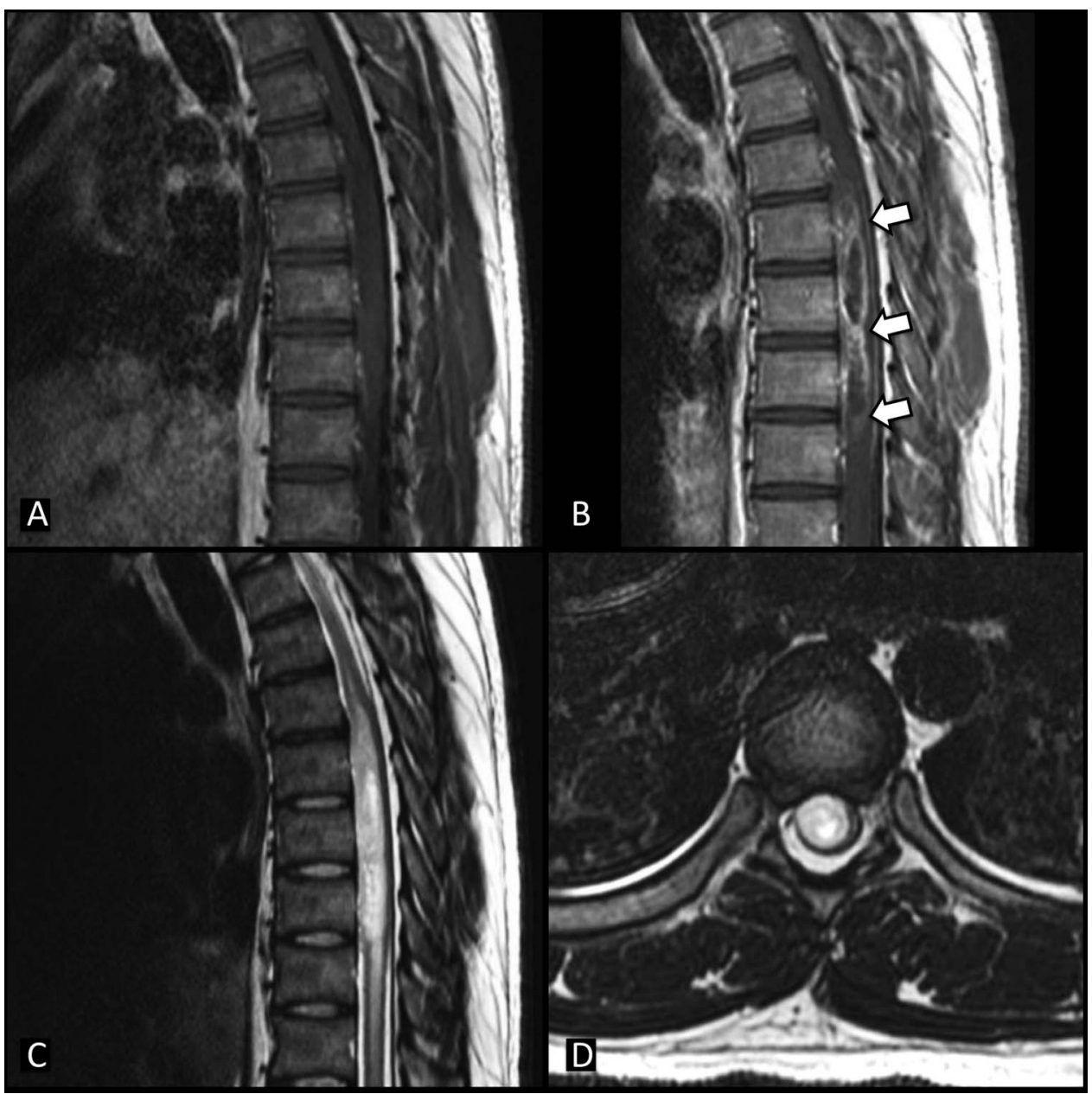

Figure 2 Astrocytoma. (A) T1 weighted. (B) T1 weighted image with gadolinium demonstrates an ill defined heterogeneously enhancing mass (white arrows) extending over four segments. It is of high T2 signal (C) and (D) and is located eccentrically within the cord, displacing the cord posteriorly and to the left. There is no definite evidence of haemorrhage. 
differentiate from normal spinal cord. ${ }^{8}$ They are isointense to hyperintense on T2 with associated tumour cyst or syrinx being common. ${ }^{4} 9$ Contrast enhancement is vivid, haemosiderin capping may be present and focal flow voids are often seen. The characteristic angiographic finding is a densely enhancing nidus with associated dilated arteries and prominent draining veins.

\section{RARE INTRAMEDULLARY TUMOURS}

There are many other rare tumours which are found in the cord (figure 3). A detailed discussion of each is beyond the scope of this article, however, a number are worth discussing briefly.

\section{Ganglioglioma}

Spinal gangliogliomas are rare, comprising $1.1 \%$ of all spinal cord neoplasms. ${ }^{6}$ They are most frequently encountered in children, representing $15 \%$ of paediatric intramedullary neoplasms. ${ }^{3}$ They are WHO grade I or II neoplasms composed of both ganglion cells and glial elements, and are most frequently located eccentrically in the cervical region, although they often involve long segments of the cord. Their imaging features are non-specific with heterogenous T1 signal, patchy contrast enhancement and hyperintense T2 signal. $^{6}$ Calcification is common and approximately half contain tumoural cysts. ${ }^{4}$

\section{Intramedullary metastases}

Intramedullary metastases are less common than leptomeningeal metastases, occurring in approximately $0.9-2.1 \%$ of cancer patients. ${ }^{10}$ They are most frequently found in the cervical cord. Lung cancer is the most common primary tumour, accounting for approximately $50 \%$ of cases. ${ }^{10}$ One-third of patients have concomitant brain metastasis, and one-quarter have leptomeningeal metastases. ${ }^{11}$ They are typically well defined, hypointense on T1, hyperintense on T2 and demonstrate avid homogeneous enhancement. Prominent oedema commonly surrounds the tumour nodule. In contrast with primary intramedullary neoplasms, associated cysts are rare. Intramedullary metastases generally occur in the setting of advanced disease and, as such, are rarely the presenting lesion. They are not surprisingly associated with a poor prognosis, mostly related to systemic disease. ${ }^{4}$

\section{Primary intramedullary lymphoma}

Lymphoma of the spinal cord is uncommon, accounting for $3.3 \%$ of all CNS lymphoma, which constitutes only $1 \%$ of all

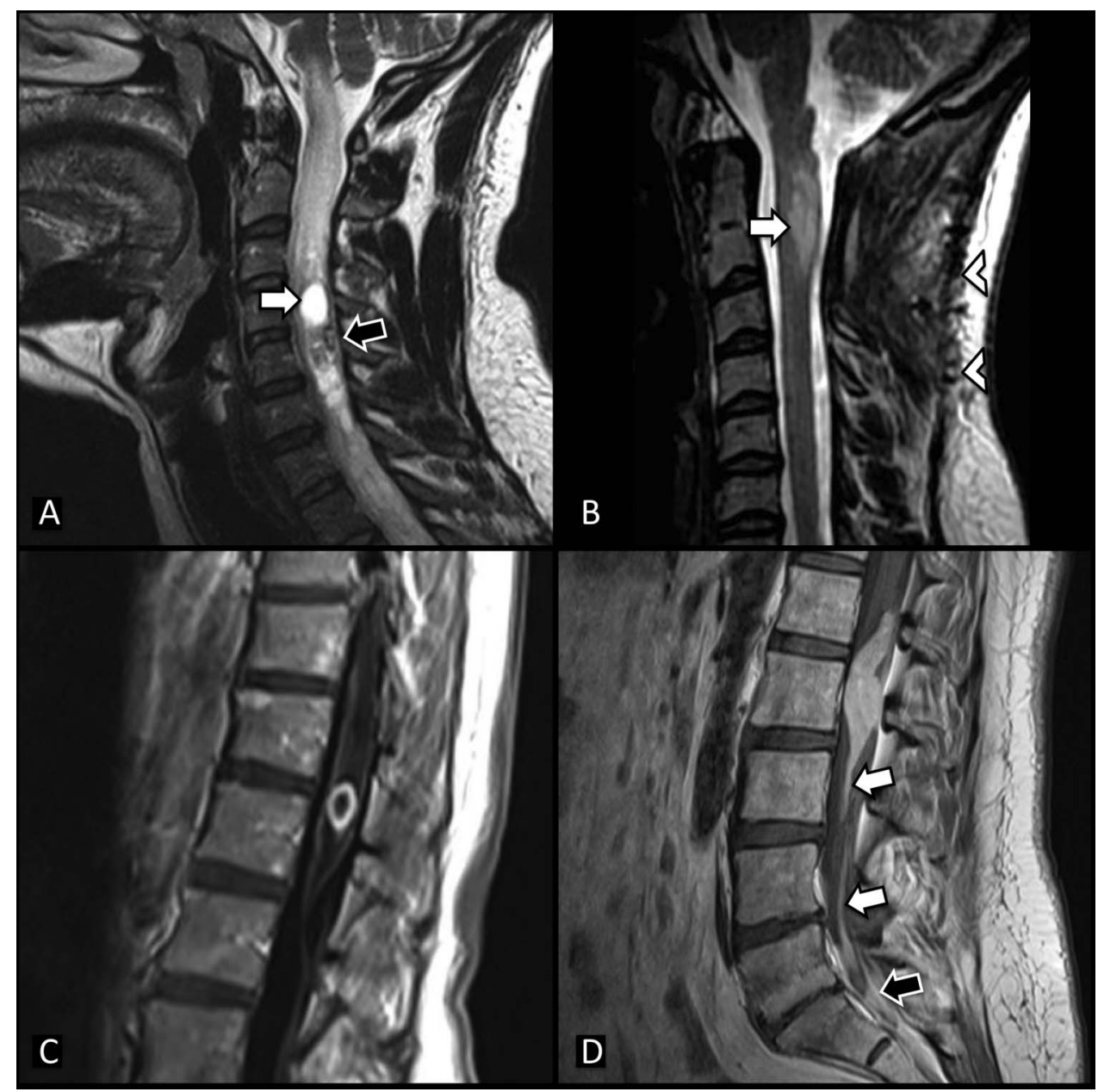

Figure 3 Rare intramedullary tumours. (A) Hemangioblastoma; T2 weighted image demonstrates peritumoural cysts (white arrow), posterior serpiginous flow void (black arrow) and cord oedema. (B) Ganglioglioma; T2 weighted image demonstrates an eccentrically located hyperintense mass (white arrow). Evidence of prior surgery for a biopsy is noted posteriorly (white arrow heads). (C) Intramedullary metastasis; a ring-enhancing lesion is demonstrated within the conus on postgadolinium T1 weighted image. Extensive cord oedema is seen as low signal on this image and confirmed on T2 weighted images (not shown). (D) Primary intramedullary lymphoma; postgadolinium T1 weighted image demonstrates a large enhancing mass with thickening and enhancement of the cauda equina (white arrows) and enhancing tissue in the thecal cul-de-sac (black arrow). 
lymphomas in the body. ${ }^{4}$ Eighty-five per cent are non-Hodgkin lymphomas. ${ }^{1}$ Most are solitary lesions located within the cervical region, however, there may be multiple lesions throughout the cord. Cord expansion is usually present, and lesions are generally poorly defined, isointense on T1 with homogeneous contrast enhancement and, in contrast to the characteristic low T2 signal intensity seen in intracranial lesions, are hyperintense on T2. Haemorrhage, tumoural cysts and syringomyelia are generally not present. ${ }^{12}$ The prognosis for patients with intramedullary spinal lymphoma is poor. ${ }^{13}$

\section{Primitive neuroendocrine tumour}

The majority of spinal primitive neuroendocrine tumours (PNETs) are secondary to metastatic spread through the subarachnoid space from a primary intracranial tumour, although cases of primary spinal PNETs have been reported. Unlike intracranial PNETs, those involving the spine are slightly more common in adults than in children. ${ }^{14}$ The lesions are usually located in the region of the filum terminale and cauda equina, are hypointense on T1, hyperintense on $\mathrm{T} 2$ and demonstrate heterogeneous enhancement. CSF seeding may produce leptomeningeal enhancement. Spinal PNETs are associated with a poor prognosis. ${ }^{4}$ Dissemination through the CSF may produce
Box 2 Intradural extramedullary tumours $(80 \%$ in adults, $65 \%$ in children)

Schwannoma (30\%)

- Meningioma (25\%)

- Neurofibroma

- Paraganglioma

- Leptomeningeal metastasis

secondary intracranial deposits; distant metastatic spread may occur to lungs, bone and lymph nodes.

\section{Solitary fibrous tumour}

Solitary fibrous tumours (SFTs) are rare spindle-cell neoplasms of probable mesenchymal origin. Although most intradural spinal SFTs are intramedullary, intradural extramedullary lesions may occur. ${ }^{15} 16$ The majority of cases are considered benign, however, malignant CNS SFTs have also been reported. ${ }^{16}$ The lesions are usually well circumscribed and encapsulated, isointense to hypointense on $\mathrm{T} 1$, and demonstrate avid homogeneous enhancement. ${ }^{17}$ Marked hypointensity is demonstrated

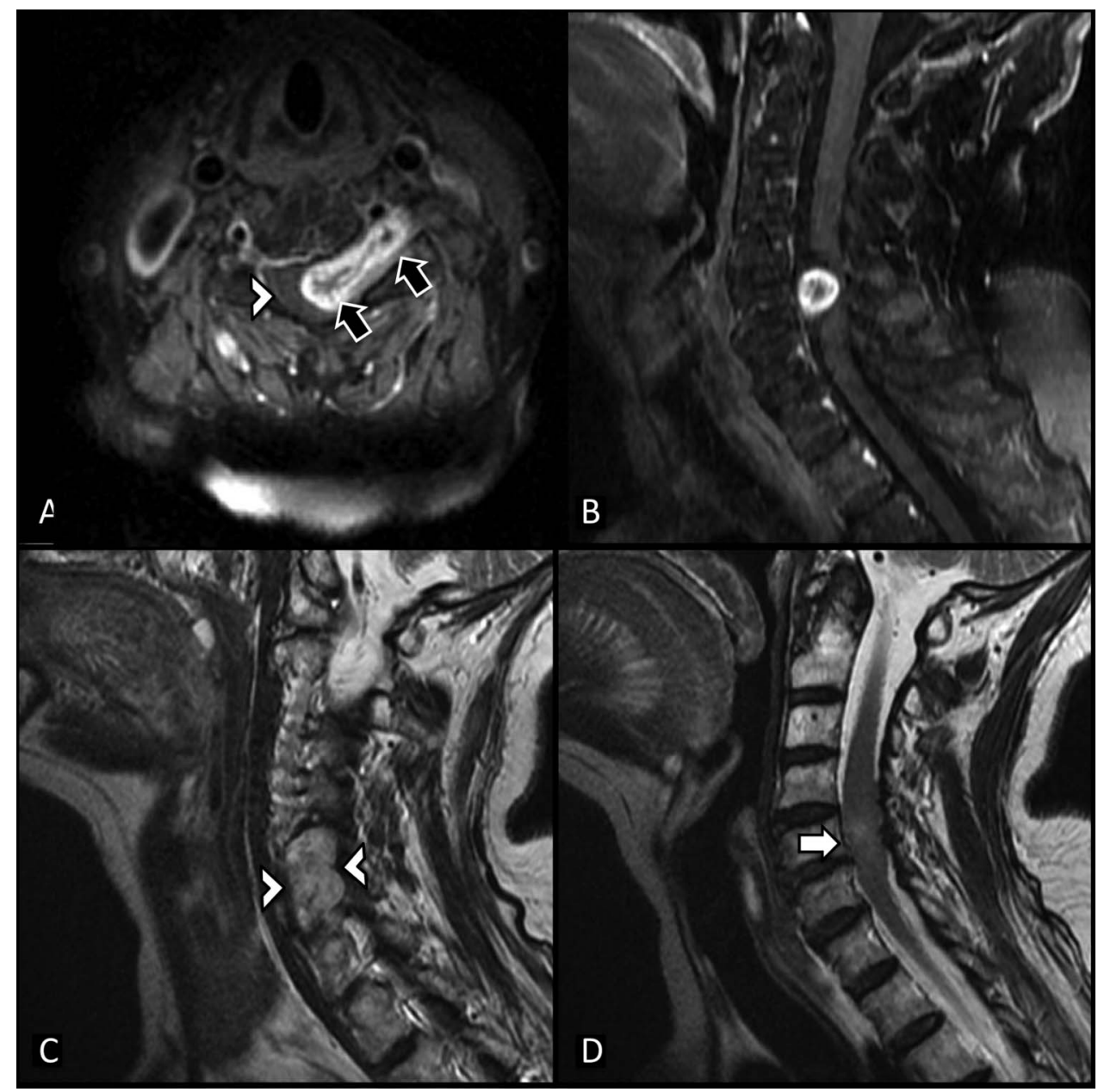

Figure 4 Dumbbell-shaped schwannoma. (A) and (B) Fat-saturated postgadolinium T1 weighted images demonstrate a vividly enhancing dumbbell shaped mass (black arrows) which passes out of the neural exit foramen. The cord is compressed and displaced towards the right (white arrow head). $T 2$ weighted images demonstrate marked expansion of the neural exit foramen by the hyperintense mass (white arrow heads) (C) and faint increased signal within the cord substance (white arrow) (D). 


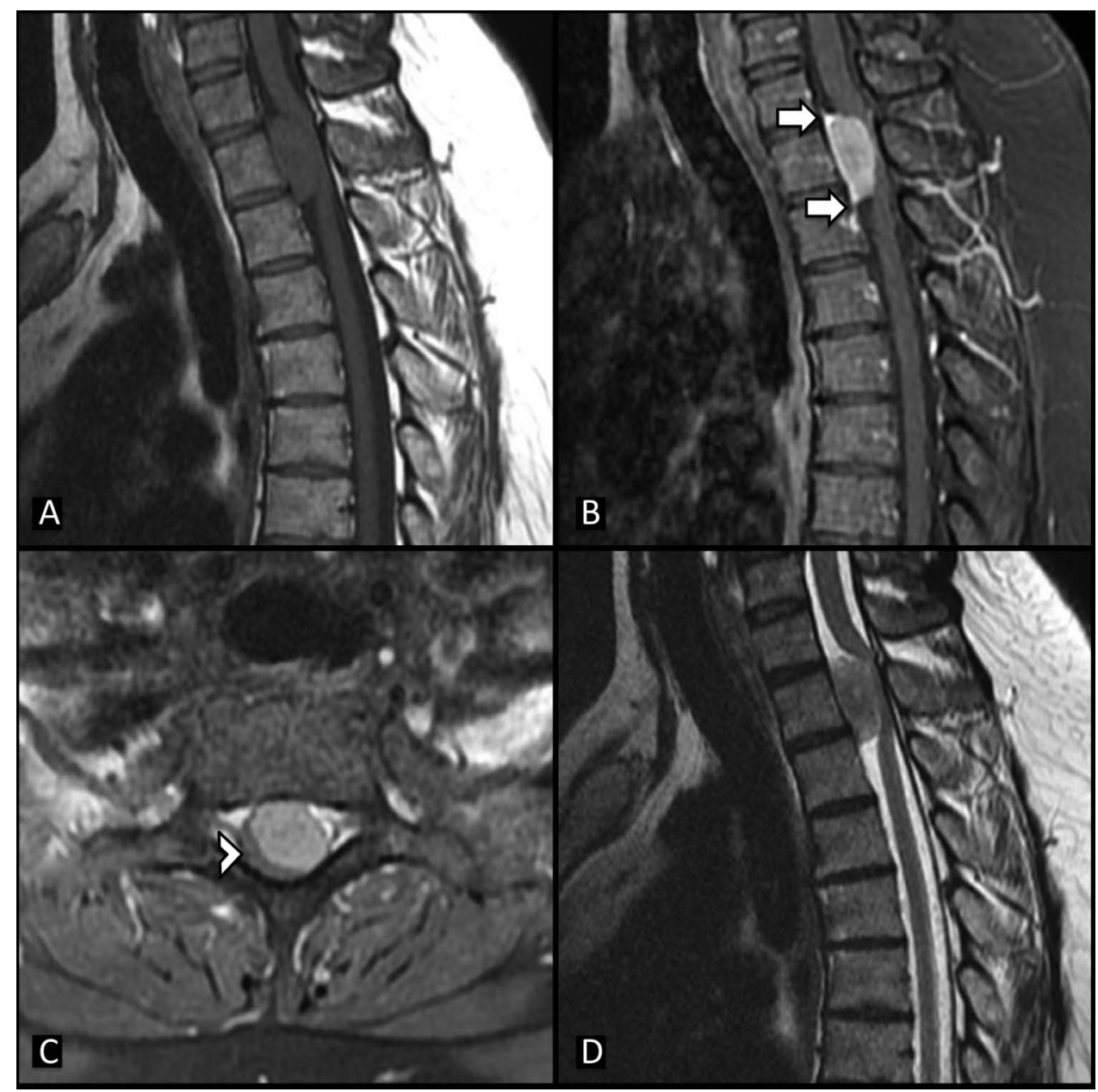

Figure 5 Meningioma. (A) T1 weighted image demonstrates a well-circumscribed intradural extramedullary mass that is isointense to the spinal cord. (B) and (C) Fat-saturated postgadolinium T1 weighted images; the mass demonstrates homogenous enhancement and a broad dural base. Dural tails are seen extending above and below the mass (white arrows). The spinal cord is markedly compressed (white arrow head). (D) The mass is slightly hyperintense on $\mathrm{T} 2$ weighted image.

on T2, a feature which helps distinguish SFTs from other spinal cord tumours, and which is thought to be due to the presence of abundant collagen fibres. ${ }^{18}$ While surgical resection is curative in most cases, recurrence has been reported.

\section{INTRADURAL EXTRAMEDULLARY TUMOURS}

Intradural extramedullary tumours are far more common than intramedullary tumours, representing $80 \%$ of all intradural tumours in adults and $65 \%$ of all intradural tumours in children (box 2). ${ }^{1}$

On MRI, intradural extramedullary lesions are characterised by displacement of the cord to the contralateral side and widening of the ipsilateral cerebrospinal fluid space immediately above and below the lesion.

\section{Schwannoma}

Schwannomas are the most common intradural extramedullary spinal tumours, representing 30\% of such lesions and occurring at a rate of approximately $0.3-0.4$ cases per 100000 persons per year. ${ }^{1} 19$ They are WHO grade I tumours and usually arise from the dorsal sensory roots. They are most frequently seen in the cervical cord but may also occur in the cauda equina region, where they are the second most commonly encountered tumour after myxopapillary ependymomas. The majority are solitary and sporadic, however, there is an association with NF2.

Surgery is the treatment of choice and gross total resection is usually curative, although in patients with NF2 there is a high incidence of new tumour formation.

\section{Radiographic features}

In general, schwannomas appear as rounded lesions. When large, they may either align themselves with the long axis of the cord, forming a sausage-shaped mass which can extend over several levels, or may protrude out of the neural exit foramen, forming a dumbbell shaped mass (figure 4). Bone remodelling may be seen, with widening of the neural exit foramen, thinning of the pedicle and posterior vertebral body scalloping. They are isointense to hypointense on T1, and hyperintense on T2, often with mixed signal. Virtually all enhance. Although they are often indistinguishable from neurofibromas, schwannomas are more frequently associated with haemorrhage, cyst formation and fatty degeneration. These findings are rare in neurofibromas. ${ }^{6}$

\section{Meningioma}

Meningiomas are the second most common intradural extramedullary spinal tumours, representing $25 \%$ of such lesions and 


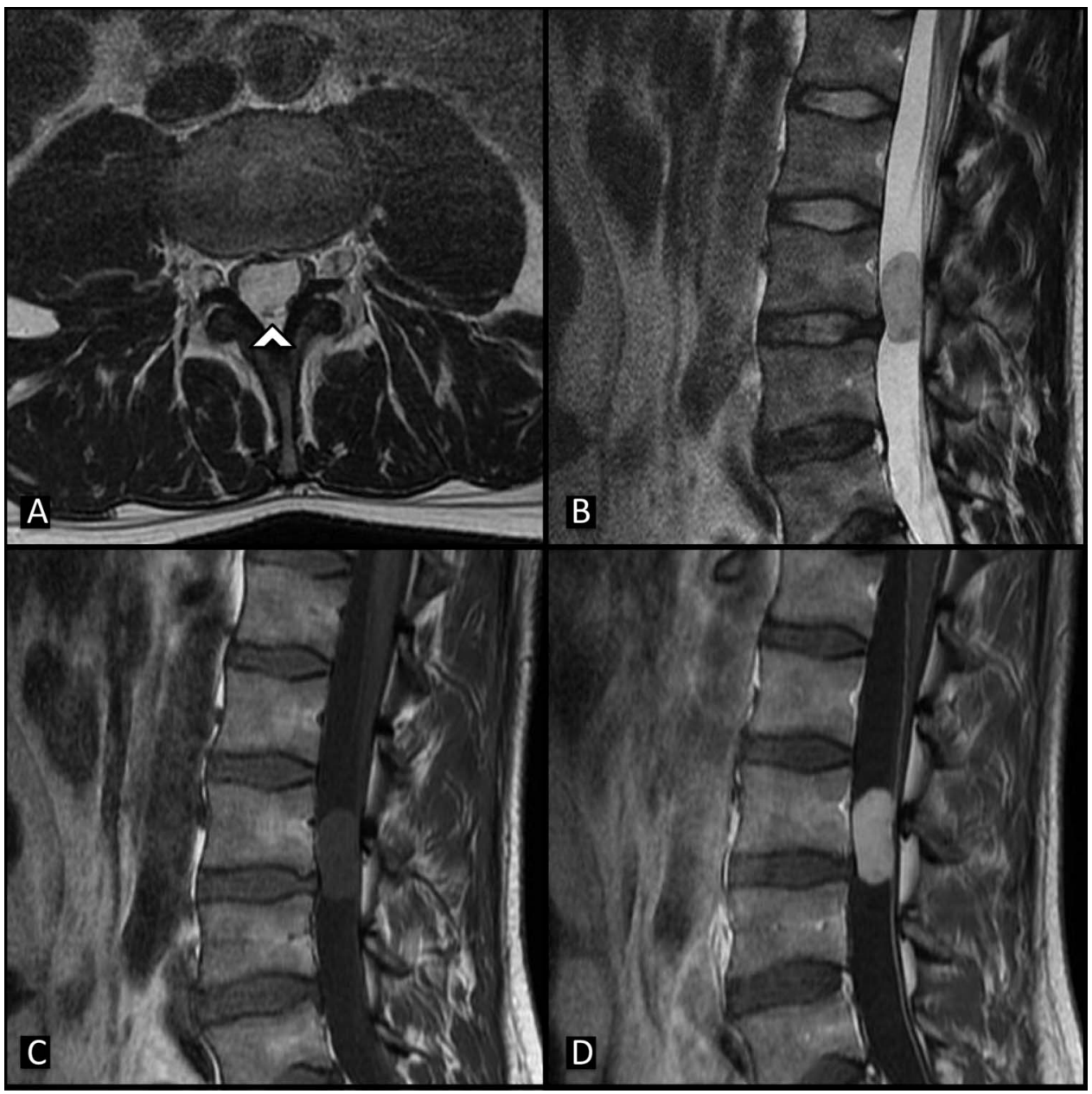

Figure 6 Myxopapillary ependymoma. (A) and (B) T2 weighted images demonstrate a central intrathecal mass in the region of the cauda equina that is of heterogenous increased signal. The filum terminale is seen running in the midline behind the mass on the axial image (white arrow head). There is near-complete effacement of the CSF space. (C) The mass demonstrates intermediate signal on the T1 weighted image and homogeneously enhances on the postcontrast image (D).

occurring in approximately 0.32 per 100000 persons per year. ${ }^{5}$ The vast majority are WHO grade I lesions. In the adult population, women are approximately 10 times more commonly affected than men, although in children there does not appear to be a sex predilection. The female preponderance in the adult population is even stronger than that associated with intracranial meningiomas, and is thought to be due to the effect of oestrogen, although the exact mechanism remains unclear. ${ }^{2021}$ Except when seen in the setting of NF2, nearly all are solitary lesions. ${ }^{1}$

\section{Box 3 Tumours of the cauda equina region}

- Myxopapillary ependymoma (90\%)

- Schwannoma

- Paraganglioma

- Metastasis

- Hemangioblastoma

- Meningioma

- Astrocytoma

- Primitive neuroendocrine tumour

- Ganglioglioma
Surgery is the treatment of choice, with complete tumour removal able to be achieved in most patients.

\section{Radiographic features}

Meningiomas are most frequently found in the thoracic region. They are often located posterolaterally in the thoracic region and anteriorly in the cervical region. ${ }^{1}$

They are well circumscribed with a broad dural attachment, and share the imaging features of intracranial meningiomas; they are usually isointense to slightly hypointense on T1 with vivid homogenous enhancement. A dural tail sign is often present (figure 5). They are isointense to slightly hyperintense on T2. Occasionally, densely calcified meningiomas are hypointense on both $\mathrm{T} 1$ and $\mathrm{T} 2$, and show only minimal contrast enhancement.

\section{Neurofibroma}

Spinal neurofibromas are WHO grade I peripheral nerve sheath tumours. There is an association with NF1. Surgery is the treatment of choice for symptomatic lesions; however, as neurofibromas tend to encase the nerve roots, they usually cannot be dissected from the parent nerve. ${ }^{9}$ Five per cent to $10 \%$ undergo malignant change, which may be indicated by rapid growth. ${ }^{22}$ 


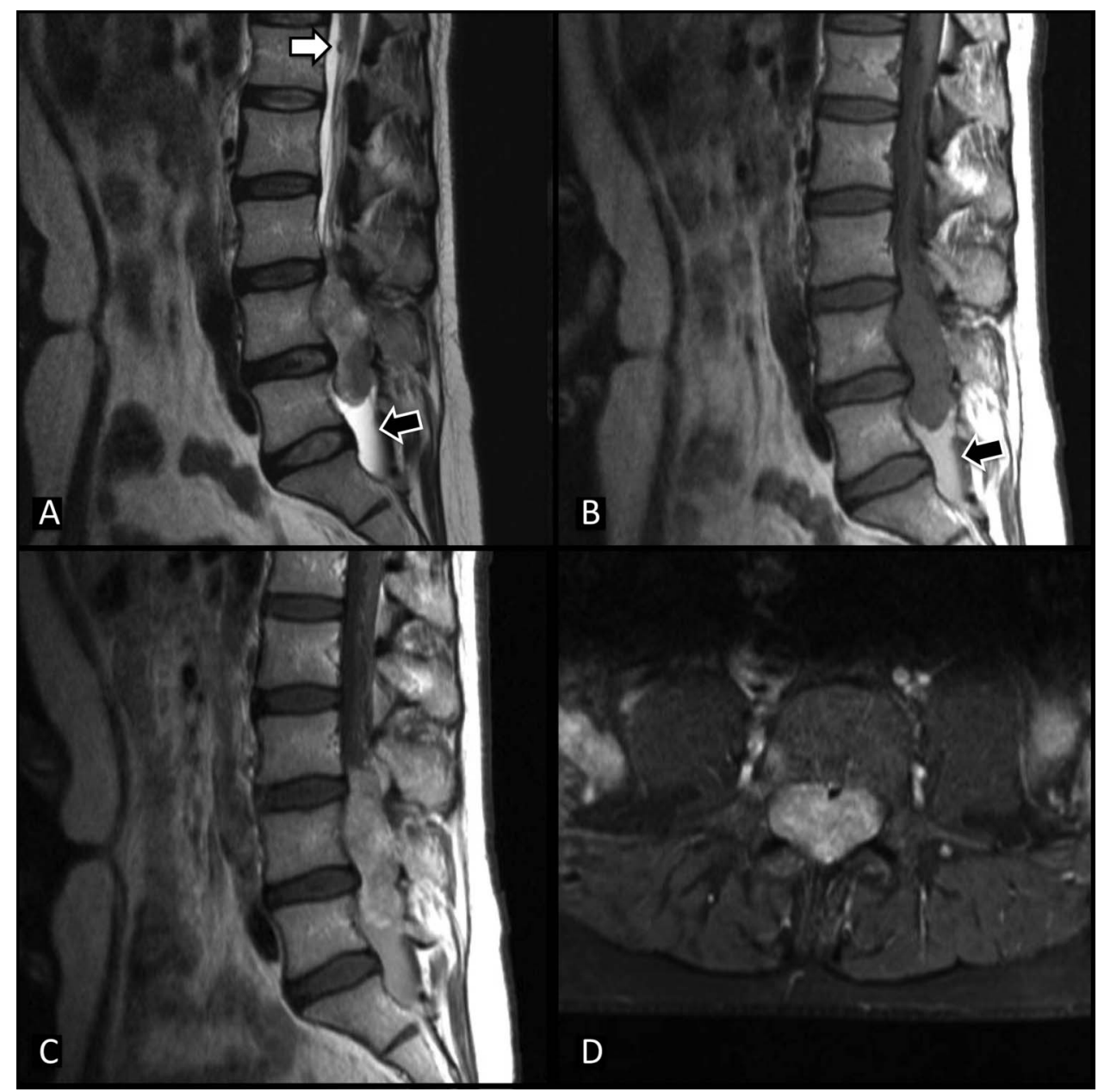

Figure 7 Paraganglioma. (A) T2 weighted image demonstrates an intermediate signal intradural extramedullary mass which causes posterior vertebral body scalloping. A prominent flow void is seen anterior to the conus (white arrow). (B) The mass also of intermediate signal on the T1 weighted image; high T1 and T2 signal fluid inferior to the mass most likely represents proteinaceous material (black arrows). (C) and (D) The mass vividly enhances postgadolinium. Spinal paragangliomas occur almost exclusively in the cauda equina region.

\section{Radiographic features}

Spinal neurofibromas are often indistinguishable from schwannomas, although a number of features may help suggest the diagnosis. They are most commonly fusiform in shape, unlike schwannomas which are characteristically round. They tend to encase the nerve roots, in contrast with schwannomas which commonly displace the nerve root due to their asymmetric growth. They are generally hypointense on T1 and hyperintense on T2, although a T2 hyperintense rim and central area of low signal ('target sign') may be seen. This sign is thought to be due to a dense central area of collagenous stroma, and although highly suggestive of neurofibroma, is occasionally also seen in schwannomas and malignant peripheral nerve sheath tumours. ${ }^{23}$ Heterogenous enhancement with areas of low signal is more characteristic of a neurofibroma than a schwannoma. As with schwannomas, bone remodelling may be seen.

\section{Myxopapillary ependymoma}

Although, strictly speaking, myxopapillary ependymomas are intramedulary tumours, they appear radiologically as extramedullary masses in the region of the cauda equina (figure 6). They are WHO grade I lesions which arise from the ependymal cells lining the inferior continuation of the central canal within the filum terminale, and represent more than $90 \%$ of tumours in the cauda equina region (box 3). ${ }^{21}$ They most commonly present in young adult men.

\section{Radiographic features}

They are usually hypointense on T1, although T1 hyperintensity is occasionally demonstrated due to a prominent mucinous component. ${ }^{24}$ They are typically hyperintense on $\mathrm{T} 2$, however, are prone to haemorrhage and, thus, often demonstrate low intensity at the tumour margins. Enhancement is virtually always seen.

\section{Paraganglioma}

Spinal paragangliomas are rare WHO grade I neoplasms that occur almost exclusively in the cauda equina region. ${ }^{25}$ Neuroendocrine symptoms are usually absent, with presentation most commonly being due to mass effect. Surgical resection is the treatment of choice, sometimes with preoperative embolisation to reduce intraoperative blood loss.

\section{Radiographic features}

Paragangliomas are usually well-circumscribed masses that are isointense on $\mathrm{T} 1$, hyperintense on $\mathrm{T} 2$ and intensely enhancing on postcontrast images. Flow voids are typically seen along the surface of and within the tumour nodule (figure 7). Haemorrhage is common, leading to a 'cap sign'. Occasionally, 


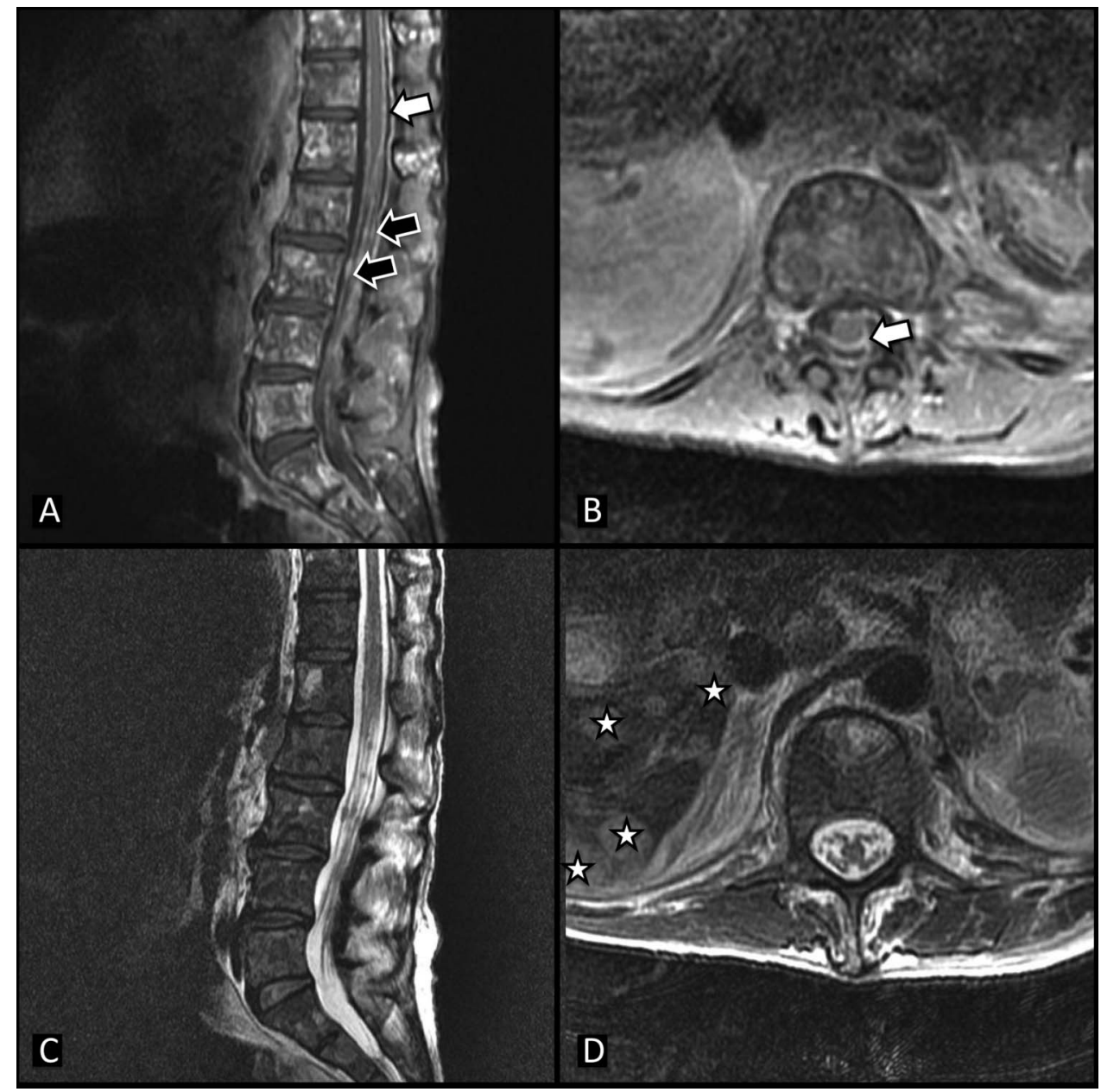

Figure 8 Leptomeningeal metastases. (A) and (B) Fat-saturated postgadolinium T1 weighted images demonstrate multiple enhancing nodules scattered along the cauda equina (black arrows) with extensive leptomeningeal enhancement of the conus (white arrows). (C) and (D) T2 weighted images; note liver (white stars) and widespread bony metastatic disease.

the characteristic 'salt-and-pepper' appearance of head and neck paragangliomas may also be demonstrated. Conventional angiography reveals an intense early blush that persists into the late arterial and early venous phases.

\section{Box 4 Lesions that mimic intradural tumours}

Intramedullary lesions

- Vascular lesions

- Cavernous malformation

- Dural arteriovenous fistula

- Spinal cord infarction

- Inflammatory lesions

- Demyelination

- Transverse myelitis

- Spinal cord abscess

- Spinal cord contusion

Intradural extramedullary lesions

- Intradural spinal lipoma

- Epidermoid cyst

- Dermoid cyst

- Neurenteric cyst

- Arachnoid cyst

\section{Leptomeningeal metastases}

Although intradural extramedullary metastases are rare, overall, they are the most common intradural extramedullary neoplasm in children. In the paediatric population, leptomeningeal metastases usually result from primary brain tumours ('drop metastases'); in adults, non-CNS tumours are more frequently encountered. The most commonly affected site is the lumbosacral spine, and multiple lesions are often seen. Prognosis depends on the primary tumour but is generally poor.

\section{Radiographic features}

T1 weighted images may demonstrate thickened nerve roots or nodular lesions that are isointense with the spinal cord. Cord oedema may be seen with more extensive disease, especially if there is an intramedullary component. Contrast-enhanced images reveal enhancing tumour nodules on the spinal cord, nerve roots or cauda equina. Innumerable small enhancing nodules may also be seen along the spinal cord and nerve roots ('sugar coating') (figure 8).

\section{LESIONS THAT MIMIC INTRADURAL TUMOURS}

Various lesions may mimic intradural tumours (box 4). The differential diagnosis of intramedullary tumours includes vascular lesions, such as cavernous malformations, dural arteriovenous fistulas and spinal cord infarction, inflammatory disorders, such as demyelination, transverse myelitis and spinal cord abscesses, and spinal cord contusions (figure 9). The differential diagnosis 


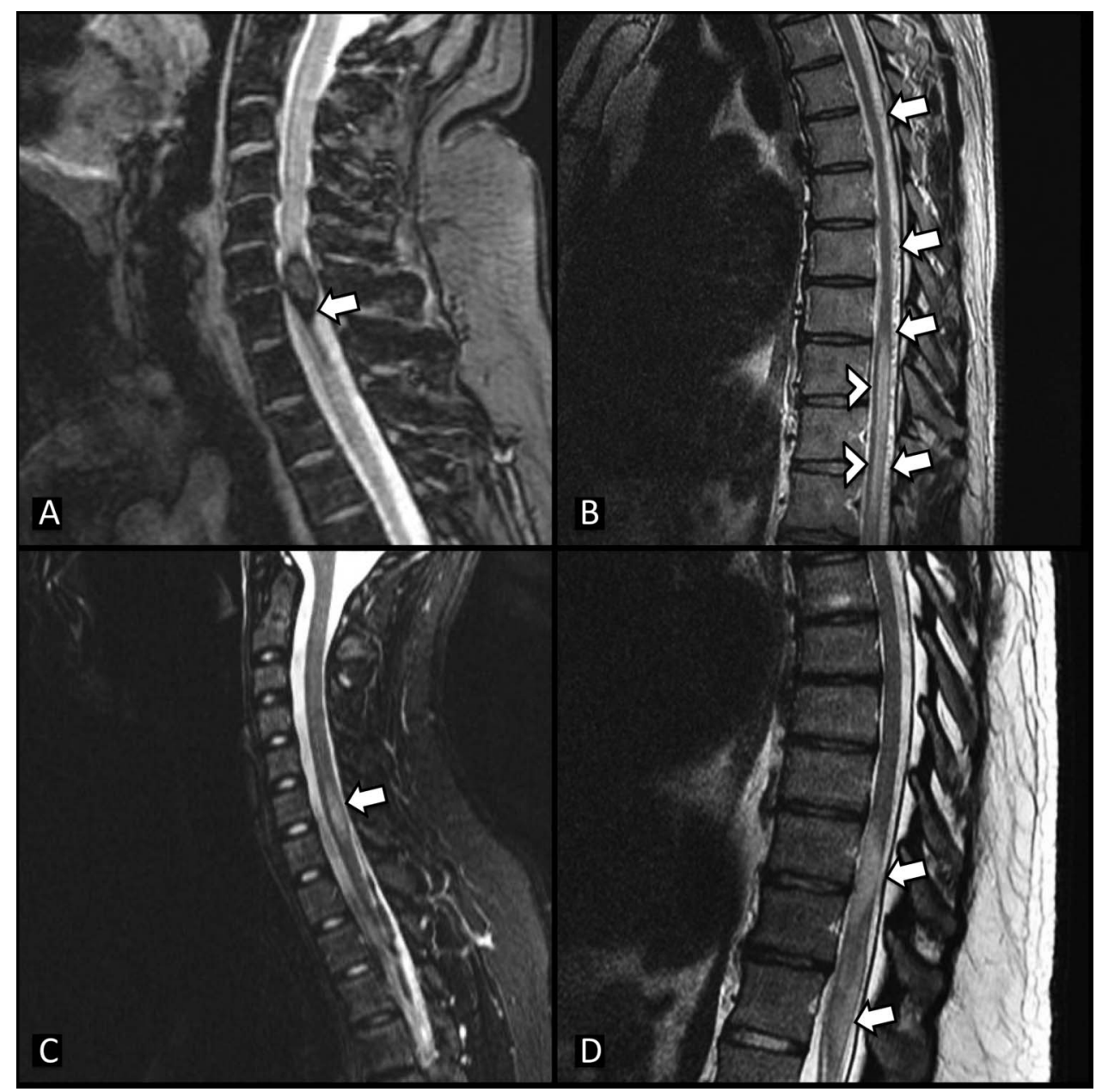

Figure 9 Lesions that mimic intramedullary tumours. (A) Cavernous malformation; gradient echo image demonstrates characteristic blooming of the haemosiderin ring (white arrow). Cavernous malformations typically demonstrate heterogenous signal on T1 and T2 weighted images due to blood products of varying ages. (B) Dural arteriovenous fistula; T2 weighted image demonstrates increased signal within the lower thoracic cord (white arrow heads) as well as numerous tortuous filling defects along the dorsal aspect of the cord (white arrows). The site of maximal MRI abnormality is not a reliable indicator of the location of the fistula; a complete spinal angiogram is therefore required. (C) Demyelination; T2 weighted image demonstrates a hyperintense lesion (white arrow) that does not cause significant spinal cord enlargement. The plaques are typically isointense to hypointense on T1 weighted images, and demonstrate variable enhancement depending on acute activity. In most patients, additional lesions of variable enhancement are present in the brain and spinal cord. (D) Transverse myelitis; T2 weighted image demonstrates increased signal and mild swelling involving most of the conus (white arrows). Lesions characteristically occupy greater than two-thirds of the cross-sectional area of the cord. An acute clinical course is typical, in contrast with that of most spinal cord tumours.

for intradural extramedullary tumours includes intradural spinal lipomas, epidermoid cysts, dermoid cysts, neurenteric cysts and arachnoid cysts (figure 10).

\section{CONCLUSION}

The differential diagnosis of intradural spinal tumours depends upon their location as intramedullary, intradural extramedullary, or related to the cauda equina/filum terminale. Although the differential is wide and there is significant overlap of the imaging appearances, a few entities make up the majority of cases. Knowledge of certain characteristics may help differentiate between lesions, and aid in preoperative planning.

\section{MULTIPLE CHOICE QUESTIONS (TRUE (T)/FALSE (F): ANSWERS AFTER THE REFERENCES)}

1. The following features are more characteristic of ependymoma than astrocytoma:

A. Scoliosis and bony remodelling

B. Eccentric location in spinal canal

C. Haemosiderin capping
D. Involvement of long cord segments

E. Ill-defined margins

2. Regarding intramedullary tumours:

A. Most patients with spinal haemangioblastomas have von Hippel-Lindau syndrome

B. Ganglioglioma typically demonstrate mixed signal intensity on T1 weighted images

C. The vast majority of patients with intramedullary metastases will have visible CNS disease elsewhere

D. Marked diffuse T2 hypointensity in an enhancing lesion is suggestive of a solitary fibrous tumour

E. Ependymomas are the most common intramedullary neoplasm in children

3. Regarding spinal nerve sheath tumours:

A. Most arise from the motor roots

B. A T2 hyperintense rim and central area of low signal ('target sign') is pathognomonic for neurofibroma

C. On postcontrast images, heterogenous enhancement with areas of low signal is more characteristic of a neurofibroma than a schwannoma 


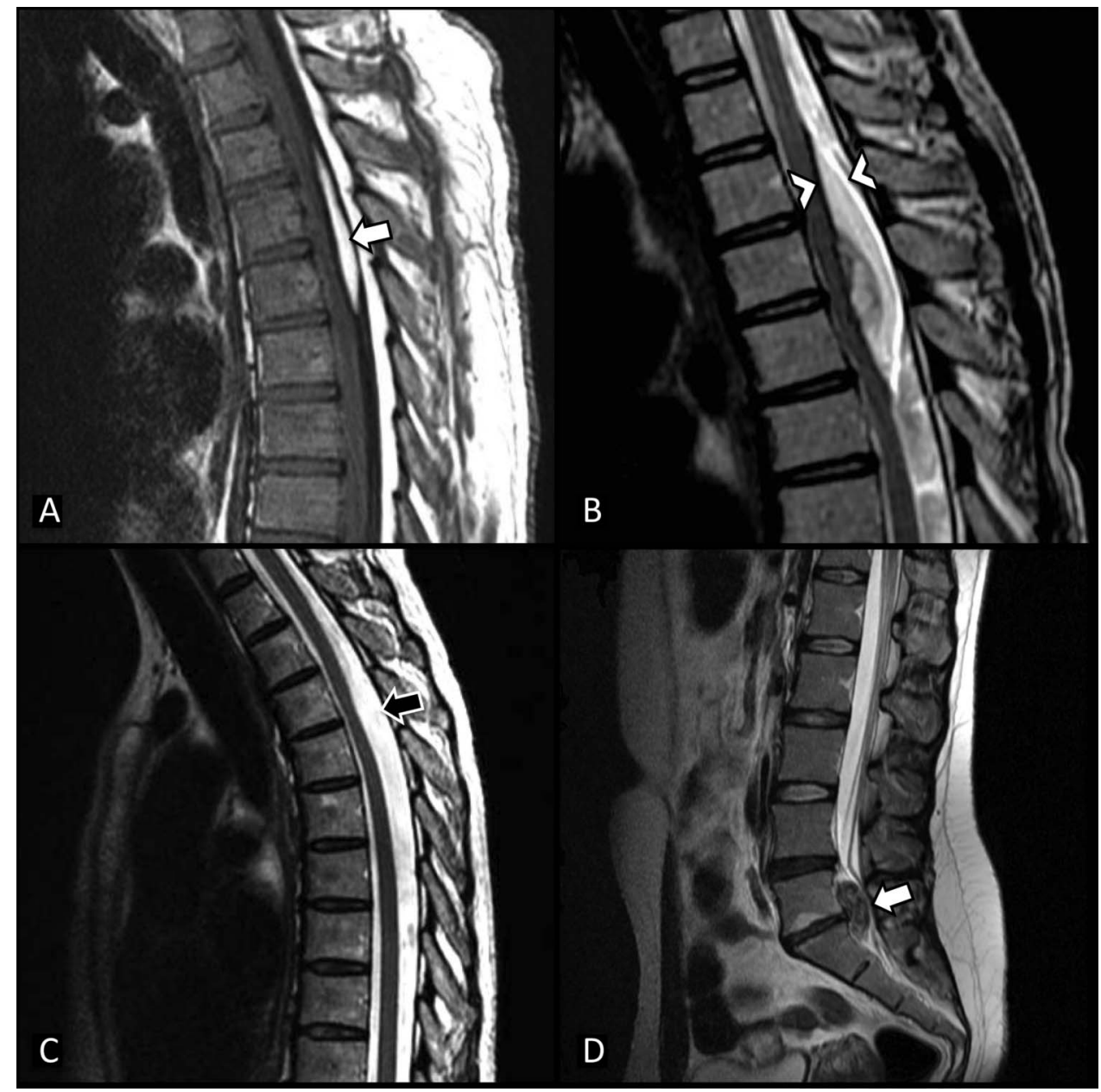

Figure 10 Lesions that mimic intradural extramedullary tumours. (A) Lipoma; T1 weighted sequence demonstrates a sharply circumscribed hyperintense mass (white arrow). The mass follows fat intensity on all sequences, does not enhance and may cause a chemical shift artefact. (B) Dermoid; T2 weighted image demonstrates a heterogenous mass posterior to the cord which contains fat and demonstrates prominent chemical shift artefact, seen as anterior dark and posterior white bounding lines (arrow heads). (C) Arachnoid cyst; T2 weighted image demonstrates anterior displacement of the thoracic cord by a mass that is isointense to CSF (black arrow). The mass follows CSF intensity on all sequences and may require myelography to distinguish it from ventral herniation of the cord. (D) Disc sequestrated fragment/extrusion; T2 weighted image demonstrates a large L5/S1 disc extrusion (white arrows). Although extradural in location, the appearance can mimic an intradural mass. Rarely calcified disc fragments can perforate the dura.

D. Schwannomas are most commonly located in the thoracic cord

E. Neurofibromas are frequently associated with haemorrhage, intrinsic vascular changes, cyst formation and fatty degeneration

4. Regarding intradural extramedullary tumours:

A. Meningiomas are the most common intradural extramedullary tumours

B. Paragangliomas usually present with neuroendocrine symptoms

C. Spinal meningiomas are more frequent in women than men

D. In the paediatric population, leptomeningeal metastases most commonly result from primary brain tumours

E. There is an increased incidence of schwannomas and meningiomas in patients with neurofibromatosis type 2

5. Regarding tumours of the cauda equina region:

A. Myxopapillary ependymomas are usually WHO grade II lesions

B. Astrocytomas are frequently located in the cauda equina region

C. Myxopapillary ependymomas most commonly present in young adult men
D. Spinal paragangliomas occur almost exclusively in the cauda equina region

E. Paragangliomas may demonstrate a 'cap sign'

\section{Main messages}

- The differential diagnosis of intradural spinal tumours depends upon their location as intramedullary, intradural extramedullary, or related to the cauda equina/filum terminale

- $95 \%$ of intramedullary tumours are glial tumours

- Spinal nerve sheath tumours and meningiomas comprise the vast majority of intradural extramedullary tumours

- Although many of the imaging characteristics of intradural tumours overlap, some features may strongly suggest one diagnosis over others

- Lesions that may mimic intradural spinal tumours include inflammatory processes, cysts, benign tumour-like masses and vascular malformations 


\section{Directions for further research}

- Adapting routine advanced imaging techniques used in the brain for the spinal cord (diffusion, perfusion, PET/CT)

- Using diffusion tensor imaging (DTI) tractography to identify the relationship of a mass to white matter tracts to enable safer and more complete surgery

\section{Key references}

- Koeller KK, Rosenblum RS, Morrison AL. Neoplasms of the spinal cord and filum terminale: radiologic-pathologic correlation. Radiographics 20(6):1721-49.

- Abul-kasim K, Thurnher MM, Mckeever P, et al. Intradural spinal tumours: current classification and MRI features. Neuroradiology 2008;50(4): 301-14.

- Smith $A B$, Soderlund KA, Rushing EJ, et al. Radiologic-pathologic correlation of paediatric and adolescent spinal neoplasms: Part 1, Intramedullary spinal neoplasms. AJR Am J Roentgenol 2012;198(1):34-43.

- Soderlund KA, Smith AB, Rushing EJ, et al. Radiologic-pathologic correlation of paediatric and adolescent spinal neoplasms: Part 2, Intradural extramedullary spinal neoplasms. AJR Am J Roentgenol 2012;198(1):44-51.

- Wald JT, Imaging of spine neoplasm. Radiol Clin North Am 2012;50(4):749-76.

Contributors SW and FG contributed to the writing of the article and share responsibility for the finished article.

Competing interests None.

Provenance and peer review Commissioned; externally peer reviewed.

\section{REFERENCES}

1 Abul-kasim K, Thurnher MM, Mckeever P, et al. Intradural spinal tumours: current classification and MRI features. Neuroradiology 2008;50:301-14.

2 American Institute of Ultrasound in Medicine. American College of Radiology; Society for Pediatric Radiology; Society of Radiologists in Ultrasound. J Ultrasound Med 2012;31:155-64 (22215784).

3 Smith AB, Soderlund KA, Rushing EJ, et al. Radiologic-pathologic correlation of pediatric and adolescent spinal neoplasms: Part 1, Intramedullary spinal neoplasms. AJR Am J Roentgenol 2012;198:34-43.

4 Koeller KK, Rosenblum RS, Morrison AL. Neoplasms of the spinal cord and filum terminale: radiologic-pathologic correlation. Radiographics 2000;20:1721-49.

5 Duong LM, Mccarthy BJ, Mclendon RE, et al. Descriptive epidemiology of malignant and nonmalignant primary spinal cord, spinal meninges, and cauda equina tumors, United States, 2004-2007. Cancer 2012;118:4220-7.

6 Grossman RI, Yousem DM. Neuroradiology, the requisites. Philadelphia, USA: Mosby Inc., 2003.

7 Brant WE, Helms CA. Fundamentals of diagnostic radiology. Philadelphia, USA: Lippincott Williams \& Wilkins, 2007.

8 Baker KB, Moran CJ, Wippold FJ, et al. MR imaging of spinal hemangioblastoma. AJR Am J Roentgenol 2000;174:377-82.

9 Osborn AG. Diagnostic neuroradiology. St. Louis, USA: Mosby Inc., 1994.

10 Schiff D, O'neill BP. Intramedullary spinal cord metastases: clinical features and treatment outcome. Neurology 1996;47:906-12.

11 Pellegrini D, Quezel MA, Bruetman JE. Intramedullary spinal cord metastasis. Arch Neurol 2009;66:1422

12 Haque $\mathrm{S}$, Law M, Abrey LE, et al. Imaging of lymphoma of the central nervous system, spine, and orbit. Radiol Clin North Am 2008;46:339-61.
13 Caruso PA, Patel MR, Joseph J, et al. Primary intramedullary lymphoma of the spinal cord mimicking cervical spondylotic myelopathy. AJR Am J Roentgenol 1998;171:526-7

14 Deme S, Ang LC, Skaf G, et al. Primary intramedullary primitive neuroectodermal tumor of the spinal cord: case report and review of the literature. Neurosurgery 1997;41:1417-20.

15 Ogawa T, Moriyama E, Beck $\mathrm{H}$, et al. Solitary fibrous tumor of the thoracic spinal cord. Neurol Med Chir (Tokyo) 2005;45:371-4.

16 Metellus P, Bouvier C, Guyotat J, et al. Solitary fibrous tumours of the central nervous system: clinicopathological and therapeutic considerations of 18 cases. Neurosurgery 2007;60:715-22.

17 Pakasa NM, Pasquier B, Chambonnière ML, et al. Atypical presentations of solitary fibrous tumours of the central nervous system: an analysis of unusual clinicopathological and outcome patterns in three new cases with a review of the literature. Virchows Arch 2005;447:81-6.

18 Kawamura M, Izawa $\mathrm{K}$, Hosono $\mathrm{N}$, et al. Solitary fibrous tumor of the spinal cord: case report and review of the literature. Neurosurgery 2004;55:433.

19 Seppala MT, Haltia MJ, Sankila RJ, et al. Long-term outcome after removal of spinal schwannoma: a clinicopathological study of 187 cases. J Neurosurg 1995;83:621-6.

20 Michaud DS, Gallo V, Schlehofer B, et al. Reproductive factors and exogenous hormone use in relation to risk of glioma and meningioma in a large European cohort study. Cancer Epidemiol Biomarkers Prev 2010;19:2562-9.

21 Wiemels J, Wrensch M, Claus EB. Epidemiology and etiology of meningioma. J Neurooncol 2010;99:307-14.

22 Weissleder R, Wittenberg J MD MG, et al. Primer of diagnostic imaging. St. Louis: Mosby Inc., 2011.

23 Soderlund KA, Smith AB, Rushing EJ, et al. Radiologic-pathologic correlation of pediatric and adolescent spinal neoplasms: Part 2, Intradural extramedullary spinal neoplasms. AJR Am J Roentgenol 2012;198:44-51.

24 Kahan H, Sklar EM, Post MJ, et al. MR characteristics of histopathologic subtypes of spinal ependymoma. AJNR Am J Neuroradiol 1996;17:143-50.

25 Wald JT. Imaging of spine neoplasm. Radiol Clin North Am 2012;50:749-76.

\section{ANSWERS}

1.
A. $\mathrm{T}$
B. $F$
C. T
D. $F$
E. $F$

2.
A. $F$
B. $\mathrm{T}$
C. $\mathrm{F}$
D. $T$
E. $F$

3.
A. $F$
B. $F$
C. $\mathrm{T}$
D. $F$
E. $F$

4.
A. $F$
B. $F$
C. $\mathrm{T}$
D. $T$
E. T

5.

A. $\mathrm{F}$

B. $\mathrm{F}$

C. $\mathrm{T}$

D. $T$

E. T 